\title{
Die Fragepartikel mo des Tscheremissischen
}

Diesen kurzen Aufsatz widme ich dem Andenken des grossen Sammlers der tscheremissischen Sprache, Ödön Beke, dessen 100. Geburtstag bald, in einem Lustrum, gefeiert wird. In den 50er Jahren wurde mir die Ehre zuteil, das Rohmaterial seines tscheremissischen dialektologischen Wörterbuches zu ordnen und druckfertig zu machen. Diese Arbeit bereicherte mich mit vielseitigen und tiefen Kenntnissen auf dem Gebiet dieser Sprache. Ich will hier nicht die hervorragenden Eigenschaften des Materials loben; eines Tages wird das Werk erscheinen und für sich selber sprechen. Ich möchte bei dieser Gelegenheit dem Andenken des Meisters huldigen, indem ich aus der bisher unveröffentlichten Quelle schöpfe.

Ödön Beke überliess mir die Aufgabe, aufgrund des verzettelten Rohmaterials das semantische Feld jeder Worteinheit festzustellen und nach gewissen Prinzipien (Bedeutung, Funktion, Phraseologie) punktweise einzuteilen. Diese Einteilung wurde von ihm überprüft: gebilligt oder berichtigt. Das semantischfunktionale Feld des Pronomens mo teilte ich auf folgende Weise ein:

mo P B M U C Č JT, ma JO JP K 1. was; 2. ob? 3. was auch immer, was ... auch (JO); im Konditionalsatz: irgendetwas (UP); 4. weshalb? (UJ JO); also (UP).

Die in Klammern angegebenen Sigel weisen auf die Dialekte hin, in denen die vorhergehende Bedeutung oder Verwendungsweise auftritt. An erster Stelle finden wir die ererbte Bedeutung des uralischen Pronomens. An zweiter Stelle er- 
scheint die eigentümliche Verwendung desselben als Fragewort. Meines Wissens hat das Pronomen mo in keiner uralischen Sprache diese Funktion, die der Verwendung im Tscheremissischen entsprechen würde. Um die Herkunft bzw. das Muster dieser Fügung zu entdecken, führen wir an erster Stelle das 'Rohmaterial' an. Hier die bei Beke gefundenen spärlichen Beispiele:

CÜ koćk $\not \partial \grave{c}$-mo? K kačkjóc-ma? 'hast du gegessen?'

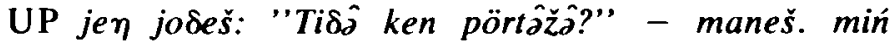
manam: "Miñan pörtem oyâl-mo?"' 'Ein Mann fragt: "Wessen Haus ist das?"' Ich antworte: "Ist das nicht mein Haus?",

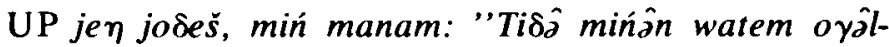
mo?'” 'Ein Mann fragt, ich antworte: 'Ist das nicht meine Frau?",

Das Fragepronomen wird auch in erweiterter Form gebraucht, z.B. CÜ moi? vgl.

CÜ pašaštâm moj àšten pâtaren ult uke, d'ot!' 'Frage, ob sie ihre Arbeit beendet haben oder nicht!'

Wir finden auch das Wort CÜ CK mojân in derselben Verwendung, z.B.:

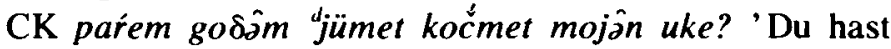
sogar am Feiertag nicht zu essen und zu trinken, was?'

Auch die Form moń ist in dieser Funktion zu finden:

JT kožlam moń ruen olt? 'sie fällten die Bäume, was?'

JT wnlanDàšt $\partial[m]$ moń üßen olt? 'sie haben das Feld bestellt, was?'

JT olaš kajen moń oyàl marec?' 'ist dein Mann nicht in die Stadt gegangen?'

JT kinDàst đjolen moń oyâl? 'brannte nicht ihr Getreide ab?'

JT jur juràn moń? 'heute hat es geregnet, was?'

JT đüaš kajen moń oyjll ońjž dök? 'Ging er nicht zum Schwiegervater zechen?'

Was uns auf den ersten Blick auffällt, ist die dialektgeographische Verteilung der "Varianten": mo und moi kommt lediglich im Dialektgebiet Uržum und Carevokokšajsk (heute JoškarOla) vor, die Form moń in einem Teil der Jaransker Region. Bisher führten wir als Ausgangspunkt nur bei Ödön Beke 
gefundene Beispiele an, jetzt möchten wir Beispielsätze aus anderen Quellen zitieren, also einen chronologischen Rückblick in die Vergangenheit werfen.

1885-86. C I! tầi patôr ulat mo? 'Eis, bist du stark?' Kuruk, tài patôr ulat mo? 'Berg, bist du stark?'

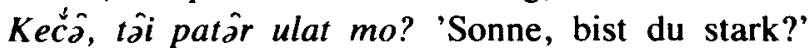
(Volmari Porkka's tscheremissische Texte. JSFOu XIII. 1895. S. 1.)

1887. P (Krasnoufimsk) Kü kuruk, šaple ulat-mo? 'Felswand, bist du stark?'

Jumo, šaple ulat-mo? 'Gott, bist du stark?' (S. 2). Tà $\hat{\partial}$-mo, torta-mo? - maneš kuy'źa 'Ist das die Fiemerstange? sagt der Alte'. (S. 11). Eigentlich: 'Dies was, Fiemerstange was?'

Awaj, tušto, tušto kum iźam ulà-mo? 'Mutter, sind meine drei Brüder dort, dort?' (S. 31 und derselbe Satz S. 32).

$T \hat{\partial} \delta \partial-\gamma n a, t \hat{\partial} \delta \hat{\partial}-\gamma n a$ üet lieš-mo? 'hast du nur so viel Butter?' (S. 36).

Pundàš wuj melnam kočkàć-mo? 'Hast du Pfannkuchen vom Baumstumpf gegessen?' (S. 37).

(Arvid Genetz: Ost-tscheremissische Sprachstudien. JSFOu VII.)

1897. Wir finden ein Zitat in Móricz Szilasis Cseremisz szótár (erschienen i.J. 1901):

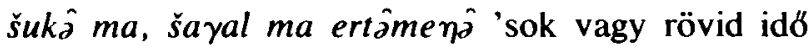
multán; nach langer oder kurzer Zeit'. - Hier bezieht sich die Fragepartikel auf die Wörter 'viel' bzw. 'wenig'. Der Satz stammt aus dem Werk O загробной жизни ... для черемисъ уездовъ Казанскаго, Царевококшайскаго, Чебоксарскаго, Уржумскаго, Яранскаго. Казань 1894.

Szilasi selber schreibt das folgende: $m a 1$. -e; fragende Partikel Bd. (d.h. Budenz NyK 4). Die Lautgestalt $m o$ als Fragepartikel kennt Szilasi nicht.'

\footnotetext{
' Szilasi irrte sich scheinbar. Die Lautgestalt mit $o$ ist bei J. Budenz vorhanden, vgl. "mo (particula interrog.) -e?/-ne? "ma B" [d.h. Bergtscheremissisch] NyK 4: 420a.
} 
1898. K -ma drückt eine Frage aus. ket-ma? 'gehst du denn?' sl'zts-ma? 'du warst ja da?' - schreibt G. J. Ramstedt (MSFOu XVII S. 76a).

Es ist vielleicht nicht uninteressant zu bemerken, dass im Märchen vom Hasen der Satz, gefunden bei Porkka und Genetz, bei Ramstedt ohne mo/ma? steht, also: ket śs stark?' (S. 197).

1905-06. U i, tajjà patâr ulat mo? 'Eis, bis du stark?' ket'ś̀, patôr ulat mo? 'Sonne, bist du stark?' (S. 157). C ket'ś̀, tôi talà ulat mo? 'id.' (S. 164).

1910. Wir finden in Wichmanns Sammlung Vierzeiler von G. Karmazin, in denen er die Fragepartikel in jeder Zeile verwendet. Der Lehrer Karmazin stammt aus dem Gouv. Ufa, Kreis Birsk, ist also ein Osttscheremisse; wir führen nur einen der Vierzeiler an:

$k \grave{u} \cdot k o m u \cdot r m=m k \grave{o} \cdot l^{\prime} t t^{\prime}{ }^{\prime} m o$ ? Hörtest du vielleicht [schon] den kuckuck rufen?

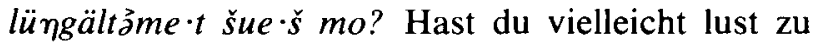
schaukeln?

menma $n$ šiń $d \check{a} a \cdot m$ mo on $d \check{z} e \cdot t$ ? Warum siehst du uns [mit unverwandtem blick] ins auge?

una $\cdot \check{s}$ nalme $\cdot t$ šue $\breve{s}$ mo? Willst du uns vielleicht $\mathrm{zu}$ gaste laden?

(Yrjö Wichmann: Volksdichtung und Volksbräuche der Tscheremissen. MSFOu LIX S. 435.) ${ }^{2}$

2 Hier eine Variante des oben im Text angeführten Liedes:

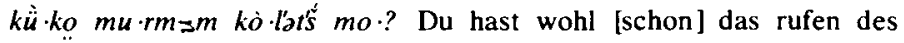
kuckucks gehört?

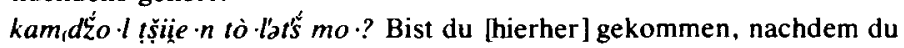
das kamisol angezogen hast?

$z \ddot{a} \eta g \ddot{a} \cdot r$ šiń $d \grave{a} a \cdot m \beta a \cdot \check{s}$ mòdòkte $\cdot n$ Indem du deine blauen augen [so] gegen [mich] spielen lässest,

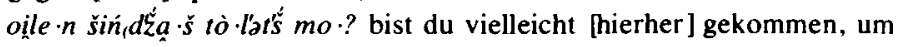
[mit mir] zu plaudern und zu sitzen.

(Y. Wichmann S. 475.)

Wir finden eine eigentümliche, beinahe spielerisch anmutende zweimalige Verwendung des Pronomens mo in dem folgenden Vierzeiler:

$k u r u \cdot k$ Bo:kten mo joškaryę? Was ist [so] rot [da] am bergabhang? 
Wir sind jetzt bei der Zeit des Bestehens der Sowjetunion angelangt. An erster Stelle teilen wir die bei Valerian Michailovitsch Vasiljev gefundenen Daten mit:

1928 (1926): $М a$ - что, мо - ли. Ужат ма - видишьли? Ма вара - что же? Что такое? (Ӱп̈̈марий = В. М. Васильев: Марий мутэр. Моско. 116а.) S. v. мо keine Beispiele.

1956. мо 2. частица вопросительная 'ли, ль'; тый толат мо? придёшь ли ты? (Марийско-русский словарь. Москва 1956. 326 ь.)

S.v. мa (312b) keine Beispiele in dieser Funktion.

1966. 1. частица вопросительная мо; придёшь ли ты? тый толат мо? . . . шутка ли? мыскара мо? (Рушла-марла мутер. Русско-марийский словарь. Москва 1966. 296.)

Diese summarischen Angaben bestätigen den bis zum heutigen Tage währenden Gebrauch des Pronomens mo als Fragepartikel. Mein Kollege Gábor Bereczki bekräftigte meine nur aufgrund von Wörterbüchern gebildete Meinung, indem er seine persönliche Erfahrung mitteilte: das Wort mo wird äusserst häufig als Fragepartikel verwendet, etwa tolat-mo?, und zwar stets mit steigender Intonation, wobei der Tongipfel auf das Wort mo fällt.

Wir wissen, dass das Pronomen $m \bullet$ in allen uralischen Sprachen ohne jegliche Suffigierung oder mit Kasusendungen versehen als Pronomen interrogativum Verwendung findet, z.B. fi. minne?, miksi?, md. E. méks?, ung. merre?, miért? etc. Es wird aber - meines Wissens - nirgends in der Funktion einer Fragepartikel wie fi. -ko/-kö?, ung. -e/-é? gebraucht. Diese Funktion muss es sich im Sonderleben der tscheremissischen Sprache angeeignet haben. Dabei sind zwei Möglichkeiten zu berücksichtigen: entweder haben wir es mit einer inneren

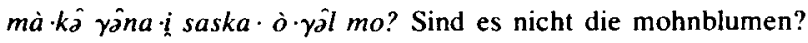

kok süryüšte $m$ mo joškaryę? Was ist [so] rot [hier] an meinen beiden wangen?

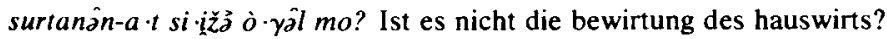

(Y. Wichmann S. 434.) 
und autochthonen Entwicklung zu tun oder die Bereicherung mit Fragefunktion ist fremdem Einfluss zuzuschreiben. Dank meiner - zwar nur sehr bescheidenen - Kenntnisse in der Turkologie bin ich geneigt, für die zweite Vermutung zu stimmen und ich werde versuchen, in den folgenden Absätzen diese Vermutung zur Gewissheit werden zu lassen.

Wir haben mit der Wirkung von zwei Türksprachen zu rechnen: mit dem Einfluss des Tatarischen und des Tschuwassischen. (Das Russische kommt nicht in Betracht, da in dieser Sprache das Pronomen interrogativum nie eine derartige Funktion erhalten hat.)

Das tatarische Pronomen milme ist in der Tat als Fragepartikel zu finden, vgl. -mi, -me -e? kérdöszócska [fragepartikel] (Gábor Bálint: Kazáni-tatár nyelvtanulmányok II. füzet. Kazáni-tatár szótár. Budapest, 1876. S. 176). Hier einige Satzbeispiele:

bar-mi? van-e? [gibt es?] (S. 170).

kilde-me? eljött-e? [ist er gekommen?] (ebenda).

kilde-mej nej? eljött-e mi (vajjon)? [ob er wohl gekommen ist?] (S. 146).

Zitate aus Texten (Kazáni-tatár nyelvtanulmányok. I. füzet. Budapest, 1875):

Boz sin batir-mi? Jég, te bátor [vagy -e? [Eis, ob du tapfer bist?] (S. 24).

Kojaš, sin batir-mi? Nap, te bátor vagy-e? [Sonne, ob du tapfer bist?] (S. 24).

Katỉn, bezgä keše kilmej-me? - digän dej. Feleség, nem jön-e valaki hozzánk? - mondta [Frau, ob nicht jemand zu uns kommt? - fragte er] (S. 26).

Küb urdiñ-mi, karčik? - dib. Sokat arattál-e, anyjuk? mondta [Hast du viel geerntet, Mütterchen? - sagte er] (S. 24).

Sin öjdä utirirya kurikmijsinn-mig? - digännär dej. Te a házban maradni nem félsz-e? - mondának [Hast du keine Angst im Hause zu bleiben? - sagten sie] (S. 27).

Ariu-mi a yaj? - dib. Hogy van, bátya? - mondta [Wie geht's, Bruder? - sagte er](S. 36, vgl. Szótár S. 10). 
Die Fragepartikel -mil-me wird auch in der heutigen tatarischen Sprache gebraucht. Diesmal begnügen wir uns mit der Anführung der Feststellung einer tatarischen Grammatik und der Erwähnung der darin gegebenen Beispiele:

,2. -мы, -ме

Частица -мь, -ме является вопросительной частицей и прибавляется к любой части речи, например: бу агачмы? дерево ли это? Буген лекция буламы? - будет ли сегодня лекция?

\section{3. -мыни, -мени}

Частица -мыни, -мени также является вопросительной частицей, но в отличие от частицей -мь, -ме она, выражая сомнение, употребляется в значении разве?, неужели? например: буген жыьелыш булмыймыни? - разве сегодня собрание не будет? Ул килгднмени? - разве он приехал?'” (Р. С. Газизов: Татарский язык. Казань 1960. 113-4.)

',Вопросительные частицы: -мы/-ме 'ли?'; -мыни/-мени 'разве? неужели?'; синме? 'ты ли? это ты?'; улмы ? 'это он?; он ли?'; бардыгызмы? 'сходили? съездили?'; син дә барасынцыни? 'разве и ты едешь?' (Татарско-русский словарь. Москва 1966. 853.)

Dieses Fragewörtchen wird (scheinbar) im Handbuch von Nicolas Poppe gar nicht erwähnt (Tatar Manuel. UAS 25. Bloomington 1963).

Die etymologisch gleiche Fragepartikel ist auch im Tschuwassischen vorhanden, und zwar in erweiterter Form (vgl. tat. -meńi). Krueger gibt folgende Varianten an: -i-měn und -im? 'really?' (S. 171). Das $i$ ist ebenfalls eine Fragepartikel, der $-m$ Auslaut ein verkümmerter Teil des Pronomen interrogativum. Als Satzbeispiel finden wir in den Texten: šan ilse pırśśĕ-i-mĕn? (Mark IV) 'Habet ihr doch kein Vertrauen?' (S. 210). (J. R. Krueger: Chuvash Manuel. Bloomington 1961. UAS 7.)

Dieselben Elemente kehren auch heutzutage im Tschuwassischen zurück, scheinbar auf einer niedrigeren Stilstufe, denn das an zweiter Stelle zu zitierende Wörterbuch (S. 636b) führt -i-men mit der Bemerkung "Umgangssprache" an. Hier einige Beispiele: 
-им частица вопросительная 'разве'; вӑл тухса кайнӑ-им? 'разве он уехал?' (106b).

Вопросительные частицы . . -им, -и-мецн 'разве? что ли? . . . разве ты не пойдёшь?' (625) (Чӑвашла-вырӑсла словарь. Москва-Мускав 1961. 625). . . . частица волросительная разговорная ('может быть') тен, -и-мӗн. (Русско-чувашский словарь. Москва 1971. 636b.)

Diese Fragepartikel ist ein Erbe aus gemeintürkischer Periode. Dies kann auch dadurch bewiesen werden, dass sie im Osman-Türkischen vorhanden ist; zur Bekräftigung dieser Behauptung dienen folgende Beispiele:

Bakan Ingiltereye gitti mi? 'Ist der Minister nach England gefahren?'

Bakan mı Ingiltereye gitti? 'Ist der Minister nach England gefahren?' (d.h. ist es der Minister, der nach England gefahren ist?)

Yorgun mu-sunuz? 'Bist du müde?'

Kocası Ingiliz mi-dir? 'Befindet sich ihr Gatte in England?'

Vapur-u gördük, değil mi? 'Sahen wir das Dampfschiff, nicht wahr?'

Güzel, değil mi? 'Sie ist schön, nicht wahr?'

Ankaraya gitti, değil mi? 'Nach Ankara ist er gefahren, nicht wahr?'

Die Beispiele zeigen, dass das Wörtchen $m i$ sich enklitisch dém Wort anschmiegt, nach dem gefragt wird. Die Fragepartikel ist im Osman-Türkischen $\mathrm{n}$ i e betont. Betont wird die dem Fragewort vorangehende Silbe.

Jetzt können wir zum Ausgangspunkt des Aufsatzes zurückkehren. Die Fragepartikel im Tscheremissischen stellt Lehngut dar, sie wurde aus Türksprachen übernommen, denn sie gewährt ein angenehmes und leicht verwendbares Element für emphatische Fragestellung.

Es ist klar, dass die Fragepartikel des Tscheremissischen eine Übernahme des tatar. me/mi darstellt. Die Formen mit $n$ und $n$ (mojen, moń) verdanken ihre Existenz dem Einfluss des Tschuwassischen (vgl. mën). Ich vermute, es handelt sich nicht 
um eine direkte Übernahme der türk. Pronomina, sondern um die Ausstattung schon vorhandener tscheremissischer Fürwörter mit Fragestellung-Funktion, also Bereicherung des semantisch-funktionalen Feldes von tscher. mo. Auf diese Weise erwarb die tscheremissische Sprache ein Mittel zur positive n Fragestellung, und zwar ein sehr bequemes Mittel, das sich gründlich von den $\mathrm{n}$ e g a $\mathrm{t}$ i v e $\mathrm{n}$ Umschreibungen anderer Sprachen, wie dem nicht wahr? des Deutschen, dem n'est-ce pas? des Französischen oder dem не правда ли? des Russischen unterscheidet.

Wenn man mir die Frage stellen würde, welche Methode des Sprachvergleichs hier verwendet wurde, käme ich in Verlegenheit. Ich bin der Auffassung, dass hier lediglich Kontaktfragen berührt wurden und sie fallen nicht unter die Rubrik des linguistischen Vergleichs. Die oben vorgeführte Erscheinung kann weder historisch-komparativ noch areal genannt werden. Sie stellt keine autochthone Charakteristik des Tscheremissischen dar, darf also nicht typologisch gewertet werden. Ich wies vor kurzem auf eine Reihe semantischer und strukturaler Sprachzüge hin (MNY 72: $213 \mathrm{ff}$., 74: $76 \mathrm{ff}$., Nyr 101: $368 \mathrm{ff}$.), die nicht als areale Erscheinungen beurteilt werden können. Es handelt sich eher um isolierte Sprachfakten, die ihre Existenz einem zivilisatorisch-kulturellen Nebeneinander verdanken. ${ }^{1}$ Indem ich dieses Urteil fälle, warne ich zugleich vor einem arealen "Rausch".

JÓZSEF ERDŐDI

\footnotetext{
1 Man darf höchstens von einem konfrontativen Verfahren sprechen. Vgl. dazu Karl Horst Schmidt: Der Sprachvergleich. Innsbrucker Beiträge zur Sprachwissenschaft. Vorträge 17. Innsbruck 1977. S. 5.
} 